\title{
BMJ Global Health Scaling up kangaroo mother care in the Philippines using policy, regulatory and systems reform to drive changes in birth practices
}

\author{
Anthony Pascual Calibo, ${ }^{1}$ Socorro De Leon Mendoza, ${ }^{2}$ Maria Asuncion Silvestre, ${ }^{3}$ \\ John Charles Scott Murray, ${ }^{4}$ Zhao Li, ${ }^{4}$ Priya Mannava, ${ }^{4}$ Jacqueline Kitong, ${ }^{5}$ \\ Mark Benjamin Quiazon, ${ }^{5}$ Howard Lawrence Sobel ${ }^{4}$
}

To cite: Calibo AP, De Leon Mendoza S, Silvestre MA, et al. Scaling up kangaroo mother care in the Philippines using policy, regulatory and systems reform to drive changes in birth practices. BMJ Global Health 2021;6:e006492. doi:10.1136/ bmjgh-2021-006492

Handling editor Seye Abimbola

Received 1 June 2021 Accepted 20 July 2021

Check for updates

(C) Author(s) (or their employer(s)) 2021. Re-use permitted under CC BY-NC. No commercial re-use. See rights and permissions. Published by BMJ.

${ }^{1}$ Disease Prevention and Control Bureau, Department of Health, Manila, Philippines

${ }^{2}$ President, Kangaroo Mother Care Foundation, Manila,

Philippines

${ }^{3}$ President, Kalusugan ng MagIna (Health of the Mother and Child), Quezon City, Philippines ${ }^{4}$ Maternal, Child Health and Quality and Safety, World Health Organization Regional Office for the Western Pacific, Manila, Philippines

${ }^{5}$ Maternal Child Health and Nutrition, World Health Organization, Country Office for Philippines, Manila, Philippines

Correspondence to Dr Howard Lawrence Sobel; sobelh@who.int

\section{ABSTRACT}

The WHO recommends kangaroo mother care (KMC) for stable preterm and low birthweight babies because it has been demonstrated to reduce mortality by up to half compared with conventional incubator-based care. Uptake of KMC in low/ middle-income countries has been limited, despite its suitability for low-resource environments. This paper reviews factors that contributed to the adoption and expansion of KMC in the Philippines. Early introduction began in 1999 but national scaleup was slow until 2014 after which a significant improvement in national adoption was observed. The proportion of target hospitals implementing KMC rose from $3 \%$ to $43 \%$ between 2014 and 2019, with 53\% of preterm and low birthweight babies receiving $\mathrm{KMC}$ by the end of this period. Expansion was led by the government which committed resources and formed partnerships with development partners and non-governmental organisations. Scale-up of KMC was built on the introduction of evidence-based newborn care practices around birth. Practice changes were promoted and supported by consensus-based policy, protocol, regulatory and health insurance changes led by multidisciplinary teams. A new approach to changing and sustaining clinical practice used hospital teams to conduct on-the-job clinical coaching and use local data to make environmental changes to support practices. Institutionalisation of early skin-to-skin contact, non-separation of mother and newborn and early initiation of exclusive breast feeding, with increased responsibility given to mothers, drove a cultural change among staff and families which allowed greater acceptance and uptake of KMC. Financial and programmatic support must be sustained and expanded to address ongoing challenges including staffing gaps, available space for KMC, willingness of some staff to adopt new practices and lack of resources for clinical coaching and follow-up.

\section{INTRODUCTION}

In the Philippines, an estimated 60000 of 1.7 million children born each year die before their fifth birthday with around half occurring among newborns. ${ }^{12}$ While child deaths decreased by $47 \%$ between 1990 and 2019, newborn mortality declined by only $26 \% .^{2}$ Along with asphyxia and sepsis, complications

\section{Summary box}

Expansion of kangaroo mother care in the Philippines was built on an effective early newborn care programme. The Department of Health led a multidisciplinary collaboration to:

- Improve the regulatory environment by incorporating updated newborn policies into preservice curricula, national licensure examinations, the health insurance reimbursement package for newborn care and licensing standards for health facilities.

- Ensure increased domestic financial support including from the Department of Health, recurrent local government unit budgets and Philippine Insurance Corporation reimbursements.

- Replace traditional training with on-the-job clinical coaching and the use of hospital newborn care teams to collect and use practice data to address systems and environmental gaps.

- Change the attitudes of staff, mothers and families by showing that early and prolonged skin-to-skin contact, breast feeding, non-separation and other evidence-based practices that could be managed easily by mothers, reduced staff workload and led to no negative consequences.

of prematurity are responsible for the vast majority of newborn deaths. ${ }^{3}$ Preterm $(<37$ weeks' gestation) and low birthweight $(<2500 \mathrm{~g})$ babies have immature immune and organ systems, low fat reserves and reduced muscle mass, placing them at a much higher risk of hypothermia, infection and death than normal birthweight babies. ${ }^{4}$ In 2014, an estimated 310193 preterm or low birthweight babies were born in the Philippines, a rate of 13.3 preterm births per 100 live births-the highest of countries with data in East Asia and the Pacific. ${ }^{5}$

Kangaroo mother care (KMC) is a method for managing preterm and low birthweight 
babies by keeping them in skin-to-skin contact on the chest of the mother (or other family members), breast feeding exclusively and providing close monitoring. ${ }^{6}$ KMC has been demonstrated to reduce mortality in small babies by up to half compared with conventional incubator-based intensive care. Other benefits include prolonged duration of exclusive breast feeding and better growth rates. ${ }^{78}$ Thus, the WHO recommends KMC for babies $\leq 2000 \mathrm{~g}$ and there have been widespread calls to introduce and implement KMC widely. ${ }^{9} 10$

KMC uptake in low/middle-income countries has been slow, despite its suitability there. ${ }^{11}$ Barriers to uptake include outdated policies and practice guidelines, distrust of mother and family-based care, concerns about safety, over-reliance on technology-based methods and staff resistance to change. ${ }^{12}$ Facility barriers include inadequate numbers of beds, space and staff skilled to provide clinical management, counselling and family support. Unsurprisingly, few countries have scaled up KMC outside isolated oases.

This practice paper reviews the experience with introducing and scaling up KMC in the Philippines. Early introduction began in 1999 but national scale-up was slow until 2014 after which a significant improvement in national adoption was observed. We reviewed existing data to identify factors associated with KMC uptake, including peer-reviewed and grey literature, national policies, programme documents and reports, and facility survey data; and a synthesis of findings of in-depth interviews with policymakers, programme managers and programme coordinators of hospitals. ${ }^{13}$ The objective was to identify and document factors important for scale-up to inform both ongoing expansion of KMC in the Philippines and introduction and scale-up in other countries in the region.

\section{EARLY INTRODUCTION USING LOCAL CHAMPIONS, BUT SLOW PROGRESS: 1999-2008}

In April 1999, the then director of the Dr Jose Fabella Memorial Hospital in Manila, the largest maternity hospital in the Philippines under the Department of Health (DoH), convinced of the benefits of KMC, sent a neonatologist and a neonatal nurse for training at the Columbia-based Fundación Canguro (Kangaroo Foundation). He then designated part of the neonatal intensive care unit as a KMC ward, and trained a KMC team consisting of nurse-midwives, paediatricians and a medical social worker. The KMC team trained intensive care staff and established a programme of regular staff training. Subsequently, the process was repeated for intensive care staff at seven hospitals in the City of Manila, with support of the national $\mathrm{DoH}$, and barangay health centre staff were trained to provide ambulatory KMC care for babies discharged from implementing hospitals. By 2005, a networking system between the Fabella Hospital and local city government health facilities was in place. In 2008, the Fabella programme coordinator established a non-governmental organisation (the $K M C$ Foundation) to support scale-up and national adoption. At the end of this period, full implementation of KMC had not progressed beyond seven hospitals in the City of Manila, where implementation was dependent on a small group of committed clinical champions.

\section{FOCUS ON IMPROVING CARE OF NEWBORNS, BUT SMALL BABIES LEFT BEHIND: 2009-2013}

A deadly hospital newborn sepsis outbreak in 2008 led to a study of childbirth practices in 51 large hospitals across the Philippines. Results showed that many evidencebased WHO recommendations were not practised and that harmful practices were common. ${ }^{14}$ In response, the DoH urgently issued a national Administrative Order and an accompanying clinical Essential Intrapartum and Newborn Care (EINC) practice protocol which included KMC for care of small babies. ${ }^{15}$ EINC comprises a package of simple evidence-based interventions shown to prevent or treat the most important causes of newborn morbidity and mortality. Interventions include: immediate and thorough drying to prevent hypothermia and stimulate breathing; immediate and sustained skin-to-skin contact after birth to prevent hypothermia, distress and hypoglycaemia and promote early and sustained breast feeding; delayed cord clamping to reduce the risk of anaemia in newborn infants and further complications in preterm infants; elimination of routine suctioning of newborns which increases the risk of apnoea and bradycardia; resuscitation for non-breathing babies; and appropriately timed hand-hygiene practices by birth attendants to reduce sepsis risk. ${ }^{16}$ EINC interventions also include the use of evidence-based criteria to limit unnecessary procedures such as caesarean sections, episiotomies and augmentation of labour, and unnecessary admissions to neonatal intensive care. ${ }^{18}$

The development of new policy guidelines was an evidence-based consultative process convened by the DoH, in collaboration with WHO, UNICEF and key non-governmental organisations working with academe, professional societies and development partners. This coalition successfully lobbied schools of midwifery, and colleges of nursing and medicine, the Professional Regulations Commission, the Health Facility and Services Regulatory Bureau, and the Philippine Health Insurance Corporation (PhilHealth) to integrate the standards into preservice curricula (2011), national licensure examinations (2011), the reimbursement package for newborn care (2011) and licensing standards for health facilities (2012), respectively (table 1$){ }^{19-22}$

EINC clinical training and quality improvement methodologies were introduced in 11 government hospitals in 2009 and subsequently expanded to all 17 regions of the country. The approach was led by government facilitators in collaboration with Kalusugan ng Mag-Ina, a local nongovernmental organisation, with support from the DoH and development partners. A job aid based on updated 
Table 1 Timeline of programme and policy inputs for the introduction and scale-up of Essential Intrapartum and Newborn Care (EINC) and kangaroo mother care (KMC), 1999-2019

\begin{tabular}{|c|c|c|}
\hline 1999-2008 & 2009-2013 & 2014-2019 \\
\hline $\begin{array}{l}\text { Dr Jose Fabella } \\
\text { Memorial Hospital } \\
\text { KMC policy } \\
\text { KMC Foundation } \\
\text { established as an } \\
\text { NGO to support } \\
\text { KMC training and } \\
\text { introduction }\end{array}$ & $\begin{array}{l}\text { Administrative Order } 2009-0025 \text { adopting new policies and protocol for essential } \\
\text { newborn care (term and preterm newborns) }(2009)^{15} \\
\text { EINC practice protocol including KMC for care of small babies and newborn care } \\
\text { clinical practice pocket guide (job aid) }(2009)^{16} \\
\text { EINC incorporated into preservice curricula and national licensure examinations } \\
(2011)^{1920} \\
\text { PhilHealth Circular 011-2011-reimbursement for newborn care package }(2011)^{22} \\
\text { EINC included in licensing standards for health facilities }(2012)^{21} \\
\text { EINC included in medium/long-term development plans and budget allocated for } \\
\text { national and local programmes (2010-onwards) } \\
\text { Communication campaigns on EINC (2010-onwards) }\end{array}$ & $\begin{array}{l}\text { Care for the small baby programme } \\
\text { established with bilateral and multilateral } \\
\text { partners and the KMC Foundation to improve } \\
\text { KMC for small babies (2014) } \\
\text { PhilHealth Circular (No. 022-2014), social } \\
\text { health insurance and benefits for women } \\
\text { about to give birth (2014) } \\
\text { PhilHealth Circular (No. 009-2017), the Z } \\
\text { benefits for premature and small newborns }{ }^{24} \\
\text { PhilHealth Circular (No. 0021-2018), } \\
\text { enhancement of newborn care package } \\
\text { (2018) }\end{array}$ \\
\hline
\end{tabular}

NGO, non-governmental organisation; PhilHealth, Philippine Health Insurance Corporation.

newborn clinical practice guidelines was developed and multidisciplinary hospital working groups made responsible for collecting data and taking action to address gaps (box 1). ${ }^{18}$

Broader institutionalisation of and demand for EINC was promoted by health professional associations who disseminated information, conducted training and increased awareness among health workers. Civil society organisations implemented social marketing activities to promote EINC and breast feeding in communities through social media and women's support groups. In addition, national government agencies, such as the Department of Social Welfare and Development, incorporated EINC into family development counselling for the poorest families. Economic planning, finance and budget ministries increased budget allocations to maternal and newborn health at national agencies and local government units, and included key standards in national medium and long-term development plans. Local government units, in turn, allocated budget lines to support facility infrastructure, equipment and supplies required for practice change at facilities.

Box 1 Systems approach to implementation of Essential Intrapartum and Newborn Care in Philippine hospitals ${ }^{18}$

On-the-job clinical coaching.

Use of clinical checklists including time-bound, step-by-step care of breathing and non-breathing babies and handwashing tasks.

- Multidisciplinary hospital teams including senior hospital managers, nursing, obstetric, neonatal, anaesthesiology staff and external experts met weekly to address barriers and solutions to evidencebased practice, focusing on revising hospital environments to enable practice change.

- Periodic clinical assessments by both internal and external review teams.

- Actions by hospital teams to address barriers to evidence-based practices and gaps identified from clinical assessments included revising hospital policies, standard operating procedures, health worker roles and physical set-ups for deliveries.

\section{SMALL BABIES GET THEIR DUE DILIGENCE: 2014-2019}

Success with the introduction of EINC for early newborn care, along with global advocacy to improve care for preterm and low birthweight babies, prompted an effort to accelerate KMC implementation. Although KMC had been adopted in the 2009 newborn care policy and protocol updates, ${ }^{1516}$ it was recognised that scale-up had been slow because policy changes had not been accompanied by any system-based efforts to secure hospital introduction. In 2013, the DoH built on the model deployed to scale up EINC, by conducting KMC training using existing EINC multidisciplinary hospital teams, periodic assessments and weekly meetings to improve hospital practices, policies and environments. Core policy, regulatory and preservice updates already made to support early newborn care included KMC for preterm and low birthweight babies and underpinned the shift towards this approach. In 2014, KMC was combined with EINC and newborn resuscitation into one initiative termed Care for the Small Baby ${ }^{23}$ Expansion of facility introduction was implemented by the KMC Foundation, in collaboration with the $\mathrm{DoH}$, and supported and financed by a multipartner consortium that included WHO and development partners. PhilHealth expanded claims for small babies (2017). ${ }^{24}$ KMC was further promoted through EINC communication channels including professional associations, civil society and the Department of Social Welfare and Development.

\section{SUMMARY OF PROGRESS}

Facilities implementing EINC rose progressively from 2010 (figure 1). By 2019, 14006 health workers had been coached in $92 \%$ ( $\mathrm{N}=1673$ ) of birth facilities. ${ }^{25}$ The number of insurance claims for the newborn care package rose from 108263 in 2011 to 1035750 in 2018, an eightfold increase over 7 years. ${ }^{26} \mathrm{KMC}$ implementation in national, regional and provincial hospitals $(\mathrm{N}=433)$ began with 13 $(3 \%)$ in 2013, rising to $60(14 \%)$ in 2015 and 186 in 2019, representing $43 \%$ of target hospitals (figure 1). ${ }^{25}$

After a baseline hospital practice study in 2008, biennial assessments of early newborn care, including KMC, 


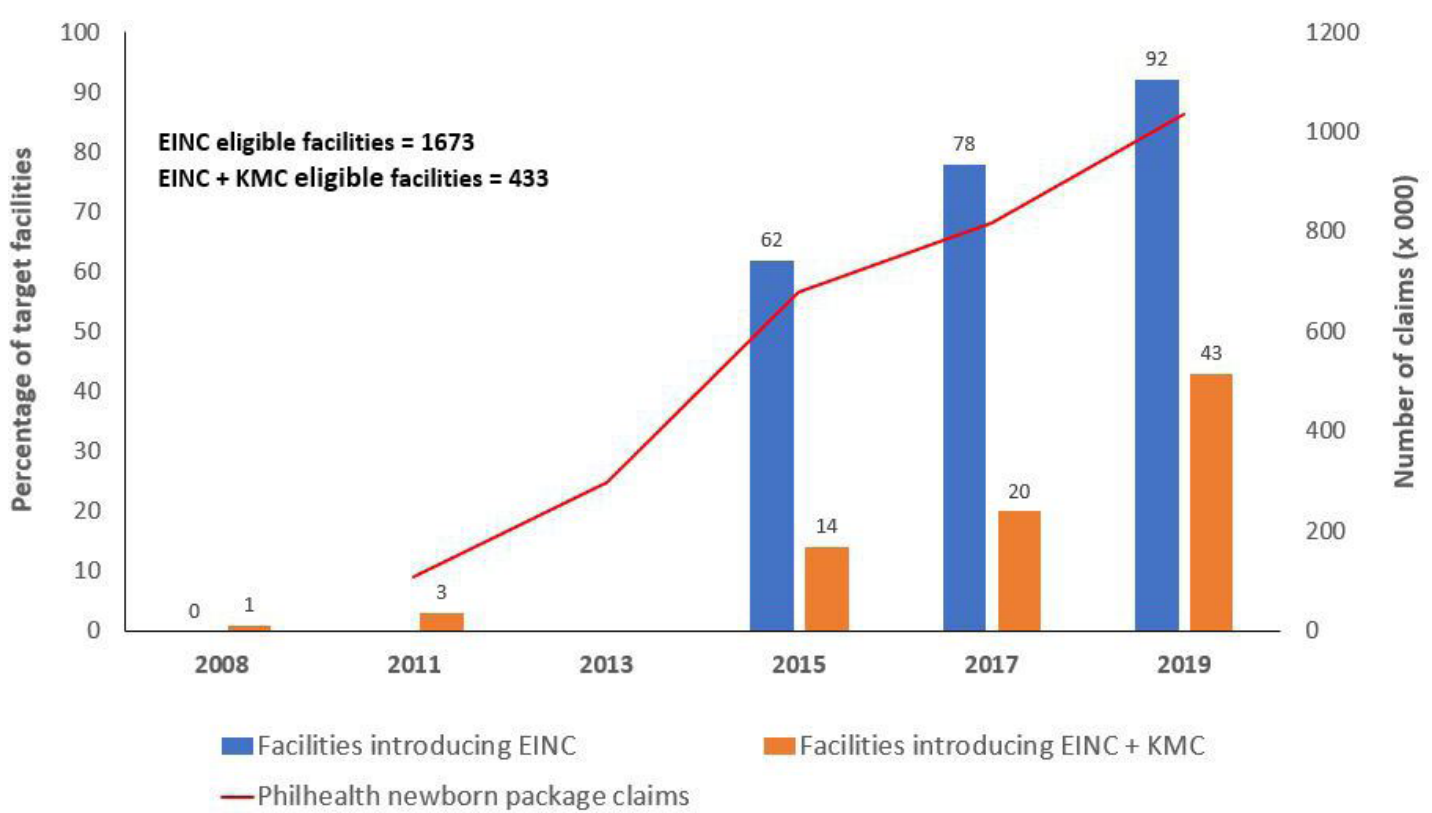

Figure 1 Facilities introducing Essential Intrapartum and Newborn Care (EINC) and kangaroo mother care (KMC) and total newborn insurance package claims, Philippines, 2008-2019. Data sources: Biennial regional EINC progress assessments, 2015, 2017 and 2019 and Philippines Insurance Corporation statistics, 2011-2018. 2526

in a sample of implementing facilities began in 2015, using standard WHO methods and tools. ${ }^{142-29}$ Data show significant progress with key early delivery and KMC practices, with improvements in immediate newborn practices preceding application of KMC uptake (figure 2). KMC was practised for $52.6 \%$ preterm and low birthweight babies by $2019 .{ }^{29}$

\section{FACTORS CONTRIBUTING TO SCALE-UP OF KMC}

Qualitative interviews programme and clinical staff found that scale-up of KMC showed a rapid rise after widespread introduction of EINC and improvements in early newborn care practices. Six common factors were identified as important for introduction.

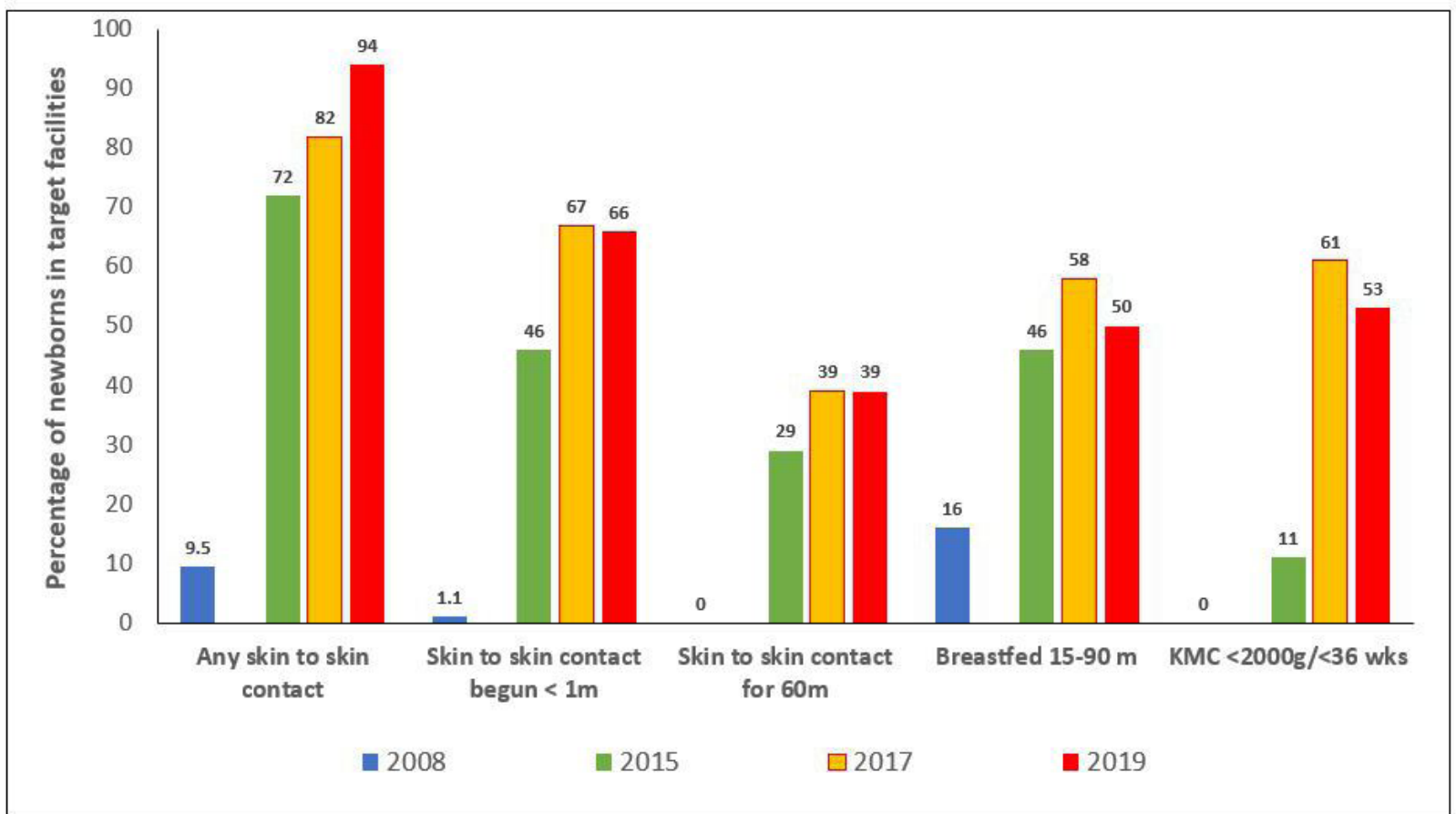

Figure 2 Facility-based estimates of early newborn care practices, Philippines, 2008-2019. Data sources: 2008: Baseline observations of 451 births at 51 hospitals ${ }^{14} ; 2015$ : 179 postpartum maternal interviews from a sample of 17 national and subnational hospitals ${ }^{27} ; 2017: 272$ postpartum maternal interviews from a sample of 28 national and subnational hospitals ${ }^{28}$; 2019: 478 postpartum maternal interviews from a sample of 45 national and subnational hospitals. ${ }^{29} \mathrm{KMC}$, kangaroo mother care. 


\section{Improve immediate newborn care policies and regulatory standards using evidence-based data}

Hospital practice data following a neonatal sepsis outbreak showed that evidence-based practices around birth were often not applied, with significant gaps in timing and sequencing of tasks that limited their effectiveness. These data were critical for highlighting gaps and securing adoption of evidence-based policies, with consensus achieved by working groups which included clinical staff, programme managers, international partners and civil society organisations. Integration of revised practice standards into preservice curricula, national licensure examinations, the reimbursement package for newborn care, and licensing standards for health facilities (table 1) was further facilitated by policy working groups which represented government stakeholders, professional associations and academic institutions. Regulatory upgrades to support evidence-based policies represented a significant shift in national commitment to improved newborn care. The increase in PhilHealth insurance claims for EINC noted from 2011 shows rapid application of the revised newborn care package. Clinical staff reported that insurance reimbursements provided an incentive to adopt evidence-based practices beyond that expected by policy upgrades alone. Updated training curricula and staff licensing standards were reported to be critical for changing staff perceptions, securing acceptance and institutionalisation of evidence-based practices in the long term; with facility regulatory standards a more important driver of shorter term uptake.

\section{Increase government financing to leverage resources from development partners and local governments}

The DoH committed financial resources for implementation costs of expanding EINC to include KMC for preterm and low birthweight babies, beginning in 2014 . The incorporation of KMC into annual implementation plans was an important signal of commitment to KMC and influenced local government planning. Resources funded introduction of clinical coaching at hospitals using non-governmental organisations, routine follow-up and supervision activities. This financial commitment was used to leverage resources from development partners (including support for the KMC Foundation which took primary responsibility for clinical coaching) and recurrent budgets of local government units. Support for the newborn package through PhilHealth insurance claims further drove financial support from local government and partners, who recognised that increased demand would require that staff and facilities were upgraded to provide KMC effectively.

\section{Go beyond training to address environmental factors that influence practice}

Introduction of KMC built on the systems approach developed for EINC. Traditional training was replaced with on-the-job clinical coaching. Newborn hospital teams monitored progress and introduced environmental changes to support practice, including reorganisation of space, patient flow, staff allocation, equipment and supplies (including KMC beds and binders) and education activities. Teams were also responsible for leveraging internal hospital resources where they were needed, and for supporting use of external resources. Active teams were able to successfully overcome barriers by engaging staff from different disciplines, senior staff and decision-makers to promote change. Previously used training methods were didactic, included limited clinical practice and often conducted off-site, requiring days away from work. Staff were positive about clinical coaching, conducted in small groups, with no presentations, lectures or reading. Benefits of coaching included that it was short duration (2 days), conducted in routine birthing environments, built on existing norms and protocols and promoted repeated practice until mastery, and therefore more likely to result in changed practice routines.

\section{Create consensus between senior maternity and neonatal care staff}

Support of national policymakers, hospital senior managers and clinical staff was critical to institutionalising KMC. Scale-up was easier when KMC 'champions' within hospitals were engaged. Engagement of this group was promoted by use of a collaborative cross-disciplinary approach to update newborn care policies and protocols between 2009 and 2011, which achieved consensus. The consensus-based approach initiated a dialogue between staff responsible for maternity and neonatal clinical care. These collaborations allowed agreement not just on evidence-based practice standards, but also on the organisation of birth environments and routines which influence whether immediate newborn care can be delivered according to standards, such as the criteria for routine suction, early bathing, mother-baby separation, neonatal intensive care admissions and application of KMC. Engagement of professional associations served to reinforce both the technical merit and political imperative of adopting new guidelines, which contributed to acceptance by policymakers. At the hospital level, lack of communication between maternity, neonatal and paediatric staff was reported to be a common reason why immediate newborn standards had not been routinely applied in the past, with each clinical discipline applying different protocols. Cross-disciplinary dialogue and agreement on practice changes required to improve care overcame some of these interdisciplinary barriers to adoption.

\section{Address staff reluctance to change old practices, concerns about the safety of small babies and fears about giving care responsibilities to mothers and other caregivers}

Changing the attitudes of staff, mothers and families towards $\mathrm{KMC}$ was built on early newborn care practices established as important for all births. Clinical introduction of EINC showed that prolonged skin-to-skin contact, early breast feeding and non-separation could 
be managed easily by mothers, reduced staff workload and led to no increases in falls or other negative consequences. Most importantly, mothers' feedback was universally positive; and this reinforcement loop incentivised staff. Community-education activities through professional associations, civil society groups and improved counselling by health staff helped normalise practices and created demand. Once this cultural shift had been established in hospital maternity and neonatal care areas, introduction of KMC-with continuous skinto-skin contact and increased reliance on the support of mothers and families-was easier to secure.

\section{Collect practice data regularly and use it to take action}

Data methods introduced for EINC were continued and expanded to include preterm and low birthweight babies. Self-monitoring was conducted by hospital teams using standard checklists, and periodically by external teams for regular monitoring at a national scale. ${ }^{60}$ Data were collected on both clinical practices and the medicines, supplies and environmental changes needed to support practices; and allowed staff to see which babies were receiving EINC and KMC practices and to understand why. Aggregated data allowed national progress to be tracked and gaps targeted for action; disaggregated hospital data allowed staff to compare their own performance with that of others, which further motivated change.

\section{CHALLENGES TO CONTINUED SCALE-UP}

Data from a sample of KMC implementing hospitals in 2019 showed that while half $(53 \%)$ of preterm and low birthweight babies received KMC, only one in five received continuous KMC (at least 20 hours with interruptions of no more than $30 \mathrm{~min}$ ) in the previous 24 -hour period. ${ }^{29}$ Moving towards continuous KMC is an ongoing challenge that will require continued monitoring and action to remove barriers to practice. Hospital staff continue to report challenges with staffing, available space, willingness of some staff to adopt new practices and with financing for ongoing activities. Staff transfers and the arrival of new staff require continuous recoaching and familiarisation with protocols and standards. Continued financial and programme support is essential to enable scale-up of KMC to non-implementing hospitals, and to continue to maintain momentum at those that have begun. A cost-effectiveness analysis is now needed to quantify total external and internal resource requirements for scale-up from all sources to allow managers to better plan future budgetary requirements. Further, this review suggests that $\mathrm{KMC}$ is less likely to be scaled up unless early essential newborn care practices are improved and sustained. Efforts to continue to improve EINC practices are therefore critical, beginning with clinical coaching supported by the formation or hospital quality teams that use simple data to address barriers to practice.

\section{CONCLUSION}

After limited progress in the 15 years since it was first introduced, KMC adoption in the Philippines improved dramatically following widespread introduction of evidence-based newborn care practices around birth. KMC was built on the consensus-based policy, protocol, regulatory and insurance changes introduced by this approach; and the introduction of a new quality improvement approach to improve early newborn care, which emphasised formation of hospital teams and use of local data to support practice through environmental changes. The effort was led by the government which committed resources and formed partnerships with development partners and non-governmental organisations. The cross-sectoral and multidisciplinary approach created consensus, made financial and other resources available and promoted wider community messaging about appropriate birth practices. Familiarity with skin-to-skin contact, non-separation and early initiation and continuation of exclusive breastfeeding practices, with increased responsibility given to mothers and families, drove a cultural change among staff and mothers which allowed greater acceptance and uptake of KMC. Financial and programmatic support must be sustained and expanded to address ongoing challenges including staffing gaps, available space for $\mathrm{KMC}$, willingness of some staff to adopt new practices and lack of resources for clinical coaching and follow-up.

Acknowledgements The authors acknowledge the support of policymakers, programme managers and programme coordinators of hospitals who provided data and other information about implementation of kangaroo mother care.

Contributors APC and HLS conceptualised the case study. ZL, PM, APC, SDLM, $\mathrm{JK}, \mathrm{MBQ}$ and JCSM conducted field interviews and collected programme data and reports. PM, ZL, JCSM and HLS conducted the data synthesis. JCSM, ZL, APC, SDLM, PM, MAS, JK, MQ and HLS drafted the manuscript. All authors reviewed and commented on the manuscript prior to finalisation.

Funding The authors have not declared a specific grant for this research from any funding agency in the public, commercial or not-for-profit sectors.

Disclaimer The authors alone are responsible for the views expressed in this article and they do not necessarily represent the views, decisions or policies of the institutions with which they are affiliated.

Competing interests All authors have completed the ICMJE uniform disclosure form at http://www.icmje.org/coi_disclosure.pdf and declare: APC was the Focal Person for the Philippines Newborn Care Program in the Family Health Office, Philippines Department of Health. SDLM is President of the KMC Foundation. MAS is President of KM; support was received from the WHO, Western Pacific Regional Office, for EINC facility assessments in 2017 and 2019; no support from any organisation for the submitted work; no financial relationships with any organisations that might have an interest in the submitted work in the previous 3 years; no other relationships or activities that could appear to have influenced the submitted work.

\section{Patient consent for publication Not required.}

Ethics approval No ethical approval was required for this article since it was a secondary analysis of available data. Ministries of Health classified the original assessments of facility-based maternal and newborn care as programmatic reviews of routine management practices of trained professionals and advised no ethical clearance was required for either the primary reviews or the secondary analysis. The assessments did not influence the time or place of deliveries or staff responsible for care, require deviations from accepted clinical practices, nor impose significant additional burden on patients or staff. Informed verbal consent was secured prior to maternal interviews, and no personal identifiers used in assessments. Feedback on assessment findings was given to facility staff and managers immediately or within 2 days of the assessment visit. 
Provenance and peer review Not commissioned; externally peer reviewed.

Data availability statement Data sharing for pure research purposes is available upon request.

Open access This is an open access article distributed in accordance with the Creative Commons Attribution Non Commercial (CC BY-NC 4.0) license, which permits others to distribute, remix, adapt, build upon this work non-commercially, and license their derivative works on different terms, provided the original work is properly cited, appropriate credit is given, any changes made indicated, and the use is non-commercial. See: http://creativecommons.org/licenses/by-nc/4.0/.

\section{REFERENCES}

1 Philippines Statistics Authority. Vital Statistics 2018 - Preliminary Counts of Births, Marriages and Deaths. Republic of the Philippines, 2019. https://psa.gov.ph/civilregistration/vital-statistics-2018preliminary-counts-births-marriages-and-deaths

2 United Nations Inter-agency Group for Child Mortality Estimation (UN IGME). Levels \& Trends in Child Mortality: Report 2020, Estimates developed by the United Nations Inter-agency Group for Child Mortality Estimation. New York: United Nations Children's Fund, 2020.

3 World Health Organization. Global Health Observatory Data Repository [website]. Geneva: World Health Organization, 2019. https://apps.who.int/gho/data/view.main.ghe3002015-CH10?lang= en

4 Lawn JE, Cousens S, Zupan J. 4 million neonatal deaths: when? where? why? The Lancet 2005;365:891-900.

5 Chawanpaiboon S, Vogel JP, Moller A-B, et al. Global, regional, and national estimates of levels of preterm birth in 2014: a systematic review and modelling analysis. Lancet Glob Health 2019;7:e37-46.

6 World Health Organization Regional Office for the Western Pacific. Introducing and sustaining EENC in hospitals: kangaroo mother care for preterm and low birth weight infants. early essential newborn care (EENC) module 4. Manila: World Health Organization, 2018.

7 Conde-Agudelo A, Díaz-Rossello JL. Kangaroo mother care to reduce morbidity and mortality in low birthweight infants. Cochrane Database Syst Rev 2016:CD002771.

8 WHO Immediate KMC Study Group, Arya S, Naburi H, et al. Immediate "Kangaroo Mother Care" and Survival of Infants with Low Birth Weight. N Engl J Med 2021;384:2028-38.

9 WHO. March of Dimes, PMNCH, Save the Children, WHO. In: Howson CP, Kinney MV, Lawn JE, eds. Born too soon: the global action report on preterm birth. Geneva: World Health Organization, 2012.

10 Nyqvist KH, Anderson GC, Bergman N, et al. Towards universal kangaroo mother care: recommendations and report from the first European conference and seventh International workshop on kangaroo mother care. Acta Paediatr 2010;99:820-6.

11 Engmann C, Wall S, Darmstadt G, et al. Consensus on kangaroo mother care acceleration. Lancet 2013;382:e26-7.

12 Chan G, Bergelson I, Smith ER, et al. Barriers and enablers of kangaroo mother care implementation from a health systems perspective: a systematic review. Health Policy Plan 2017;32:1466-75.

13 Gale NK, Heath G, Cameron E, et al. Using the framework method for the analysis of qualitative data in multi-disciplinary health research. BMC Med Res Methodol 2013;13:117.

14 Sobel HL, Silvestre MAA, Mantaring JBV, et al. Immediate newborn care practices delay thermoregulation and breastfeeding initiation. Acta Paediatr 2011;100:1127-33.

15 Philippines Department of Health, Office of the Secretary. Adopting new policies and protocol on essential newborn care. administrative order N.2009-0025. Republic of Philippines: Philippines Department of Health, 2009. https://elibrary.judiciary.gov.ph/thebookshelf/ showdocs $/ 10 / 49220$
16 Department of Health Republic of the Philippines, World Health Organization Western Pacific Region, UNICEF. Newborn care until the first week of life: clinical practice pocket guide. Manila: World Health Organization, 2009.

17 World Health Organization. WHO recommendations on newborn health: guidelines Approved by the who guidelines review Committee. Geneva: World Health Organization, 2017.

18 Silvestre MAA, Mannava P, Corsino MA, et al. Improving immediate newborn care practices in Philippine hospitals: impact of a national quality of care initiative 2008-2015. Int J Qual Health Care 2018;30:537-44.

19 Philippines Department of Health, Office of the Secretary. Department personnel order: 2011-3597. July 15, 2011. attendance to the orientation on the maternal, newborn, child health and nutrition (MNCHN) strategy and the essential intrapartum and newborn care (EINC) for the officers, board members and directors of the association of the deans of the Philippine colleges of nursing (ADPCN) and association of Philippine schools of midwifery (APSOM) on July 22, 2011 at the San Lazaro Hospital Amphitheater, DOH compound, STa. cruz, Manila. Republic of Philippines: Philippines Department of Health, 2011.

20 Philippines Department of Health, Office of the Secretary. Department personnel order: 2011-4710, August 29, 2011. attendance to the MNCHN-Essential intrapartum and newborn care (EINC) best practice forum on September 30, 2011 in Makat City. Republic of Philippines: Philippines Department of Health, 2011.

21 Philippines Department of Health, Office of the Secretary. Administrative order No. 2012-0012 - rules and regulations governing the new classification of hospitals and other health facilities in the Philippines. Republic of Philippines: Philippines Department of Health, 2012. https://hfsrb.doh.gov.ph/hospital

22 Philippines Insurance Corporation. Health circular 011-2011. New PhilHealth case rates for selected medical cases and surgical procedures and the NO balance billing policy. Pasig City, 2011. Available: https://www.philhealth.gov.ph/circulars/ 2011/circ11 2011.pdf

23 Philippines Department of Health Family Health Office. Care for the small baby program implementation review and workshop, November 17-19, 2015. Republic of Philippines: Philippines Department of Health, 2015

24 Philippines Insurance Corporation. Health circular No. 2017-0009. The $Z$ benefits for premature and small newborns. Pasig City, 2017. Available: https://www.philhealth.gov.ph/ circulars/2017/circ20170009.pdf

25 Western Pacific Regional Office of the World Health Organization (WHO/WPRO). Early essential newborn care regional database. Manila: WHO/WPRO, 2021.

26 Philippines Insurance Corporation (PIC). Stats and charts, 20112018. PIC, Republic of Philippines. Available: https://www.philhealth. gov.ph/about_us/statsncharts/\#gsc.tab=0

27 Philippines Department of Health, Kalusugan ng Mag-Ina, WHO Philippines. Annual implementation review of essential intrapartum and newborn care in the Philippines: 2015. Republic of Philippines: Philippines Department of Health, 2015.

28 Philippines Department of Health, Kalusugan ng Mag-Ina, WHO Philippines. Annual implementation review of essential intrapartum and newborn care in the Philippines: final report, July 2017. Republic of Philippines: Philippines Department of Health, 2017.

29 Philippines Department of Health, Community Medicine Development Foundation, WHO Philippines. Early essential newborn care facility assessment for the annual implementation review: final report, November 19, 2019. Republic of Philippines: Philippines Department of Health, 2019

30 World Health Organization Regional Office for the Western Pacific. Annual implementation review and planning guide (early essential newborn care, module 1). Manila: WHO, 2016. http://iris.wpro.who. int/bitstream/handle/10665.1/ 13978/9789290618362-eng.pdf 